\title{
HMGA2 promotes the migration and invasion of gallbladder cancer cells and HMGA2 knockdown inhibits angiogenesis via targeting VEGFA
}

\author{
JUN YAN, PENG DAI, XUELIANG QIN, YANPING HE and YU ZHANG \\ Department of General Surgery, Shanxi Provincial People's Hospital, Taiyuan, Shanxi 030012, P.R. China
}

Received March 29, 2021; Accepted June 17, 2021

DOI: $10.3892 / \mathrm{mmr} .2021 .12570$

\begin{abstract}
The high mobility group AT-hook 2 (HMGA2) protein has been found to be upregulated in the majority of tumor types and is associated with a poor prognosis. Previous studies have suggested the oncogenic role of HMGA2 in gallbladder cancer (GBC). The present study aimed to investigate the effects of HMGA2 on the invasion, migration and angiogenesis of GBC cells. To achieve this aim, HMGA2 was overexpressed or silenced in the GBC cell line, EH-GB1, and then the proliferation, migration, invasion and epithelial-mesenchymal transition (EMT) abilities of EH-GB1 cells were investigated using Cell Counting Kit-8, wound healing, Transwell and western blotting assays. In addition, the expression levels of VEGFA were determined in EH-GB1 cells using western blotting and reverse transcription-quantitative PCR following HMGA2 overexpression or silencing. Furthermore, HMGA2-silenced EH-GB1 cells were transfected with VEGFA overexpression plasmids to evaluate the tube formation ability of HUVECs using tube formation assay. The results demonstrated that HMGA2 silencing inhibited GBC cell proliferation, migration, invasion and EMT, as evidenced by the downregulated expression of Ki67, proliferating cell nuclear antigen, MMP2, MMP9, N-cadherin, snail family transcriptional repressor 2 and zinc finger E-box-binding homeobox 1, and attenuated cell migration and invasion. However, the opposite results were obtained following HMGA2 overexpression. Moreover, HMGA2 knockdown and overexpression downregulated and upregulated VEGFA expression, respectively. In addition, the tube formation ability of HUVECs and the expression levels of CD31, VEGFR1 and VEGFR2 were downregulated following HMGA2 silencing. However, these effects were partially rescued by simultaneous VEGFA overexpression. In conclusion, the findings of the
\end{abstract}

Correspondence to: Professor Yu Zhang, Department of General Surgery, Shanxi Provincial People's Hospital, 29 Shuangtasi Street, Taiyuan, Shanxi 030012, P.R. China

E-mail: yuzhang20@126.com

Key words: angiogenesis, gallbladder carcinoma, high mobility group AT-hook 2, migration, invasion, VEGFA present study revealed that HMGA2 may promote GBC cell migration, invasion, EMT and angiogenesis. Therefore, inhibiting HMGA2 expression could be considered as a possible therapeutic approach for GBC.

\section{Introduction}

Gallbladder cancer (GBC) is the most common malignant tumor of the bile duct system, accounting for $80-95 \%$ of all malignant biliary tract tumor cases worldwide (1). Due to the insidious onset of GBC and the lack of specific symptoms at the early stages, the majority of GBC cases are diagnosed in the middle and late stages of the disease (2). GBC is often misdiagnosed as biliary colic clinically (3). The current main treatment option for GBC is radical cholecystectomy, but the resectability of the tumor must be evaluated prior to surgery to determine eligibility. If the GBC can be resected, the liver function should be evaluated and adjuvant treatment should be administered after surgery. If the cancer is unresectable, chemotherapy or radiotherapy can be prescribed for the treatment of the disease (3). The diagnosis of cholangiocarcinoma, which is also a type of biliary tract cancer, is obtained through imaging techniques and invasive examinations, followed by pathological confirmation. These diagnostic approaches are roughly the same as those used for GBC, and so are the treatments regimens (4). For patients with advanced GBC, it is difficult to achieve the desired therapeutic effect using surgery, radiotherapy and chemotherapy $(5,6)$. Although, several molecular prognostic markers for GBC have been discovered, including KRAS, HER2, tumor protein 53 (p53) and p16, the reduced specificity and instability of these markers limit their application $(7,8)$. Therefore, the current treatment strategy for GBC is still based on surgery combined with adjuvant radiotherapy and chemotherapy (9). Until recently, the basic molecular mechanisms underlying the occurrence, development and metastasis of GBC have not been fully clarified. Therefore, further studies on these mechanisms are urgently required.

The high mobility group AT-hook 2 (HMGA2) protein is a non-histone, architectural transcription factor that modulates the transcription of numerous genes (10), including snail family transcriptional repressor 2 (Slug) (11), SRY-related HMG-box (12) and twist family bHLH transcription factor (13). It has been reported that HMGA2 regulates several biological 
processes, including cell cycle progression, DNA damage repair, apoptosis, epithelial-mesenchymal transition (EMT) and cell senescence (14). Furthermore, the upregulation of HMGA2 is a common feature of malignancy, and its increased expression has been associated with a poor prognosis and reduced chemotherapeutic efficacy in several types of cancer, such as colorectal, gastric and ovarian cancer $(10,15)$. Accumulating evidence has indicated that determining HMGA2 expression could be used as a routine procedure in clinical tumor analysis $(10,16,17)$. A previous study demonstrated that HMGA2 was upregulated in gallbladder adenocarcinoma tissues compared with adjacent normal tissues, and its expression was closely associated with a poor clinical prognosis and the metastasis of gallbladder adenocarcinoma (18). Another study demonstrated that microRNA-26a acted as a tumor suppressor by inhibiting GBC cell proliferation via directly targeting and negatively regulating HMGA2 expression (19). The aforementioned findings suggested that HMGA2 may play a role in promoting the carcinogenesis and progression of GBC. However, to the best of our knowledge, the effect of HMGA2 on promoting GBC cell invasion, migration and angiogenesis has not been previously investigated.

Metastasis is the leading cause of cancer-related mortality (20). It is estimated that $\sim 90 \%$ of all malignant solid tumors are caused by the spread and distant metastasis of tumor cells $(21,22)$. The growth of solid tumors is accompanied by the induction of angiogenesis (23). The present study aimed to investigate the effect of HMGA2 knockdown and overexpression on GBC cell invasion, migration and angiogenesis. The findings of the current article may provide a potential novel treatment approach for GBC.

\section{Materials and methods}

Cell culture and treatment. The human GBC cell line, EH-GB1 (16) was obtained from The Cell Bank of Type Culture Collection of The Chinese Academy of Sciences. Cells were cultured in DMEM supplemented with 15\% FBS (both from Gibco; Thermo Fisher Scientific, Inc.), and maintained at $37^{\circ} \mathrm{C}$ in a humidified atmosphere containing $5 \% \mathrm{CO}_{2}$. HUVECs were purchased from Clonetics ${ }^{\mathrm{TM}}$ (Lonza Group Ltd.) and cultured in DMEM supplemented with $10 \% \mathrm{FBS}$ and $1 \%$ penicillin/streptomycin at $37^{\circ} \mathrm{C}$ in a $5 \% \mathrm{CO}_{2}$ incubator. The culture medium of both cultures was replaced every 1-2 days. Once cells reached $85-90 \%$ confluence, they were passaged at a ratio of 1:3. Untreated cells were used as the control group.

For the tube formation assays and detection of CD31, VEGFR2 and VEGFR1 expression, HUVECs between passages two and five were incubated with DMEM from EH-GB1 cells transfected with different small interfering RNAs (siRNAs)/overexpression (ov) plasmids at $37^{\circ} \mathrm{C}$ for $24 \mathrm{~h}$.

Cell transfection. The ov-HMGA2 and ov-VEGFA pcDNA3.1 plasmids containing full-length sequences of the genes and the empty plasmid [used as a negative control (ov-NC)] were obtained from GenScript. siRNA targeting HMGA2 (siRNA-HMGA2; sense, 5'-CAGCCUGAAUAACUUGAA CTT-3' and antisense, 5'-GUUCAAGUUAUUCAGGCUGTT-3') and siRNA-NC (sense, 5'-UUCUCCGAACGUGUCACGUTT-3' and antisense, 5'-ACGUGACACGUUCGGAGAATT-3') were purchased from Guangzhou RiboBio Co.,Ltd. Cells were seeded into 6-well plates at a density of $2 \times 10^{5}$ cells/well and transfected with 80 pmol ov-HMGA2, ov-VEGFA, ov-NC, siRNA-HMGA2 or siRNA-NC using Lipofectamine ${ }^{\circledR} 2000$ (Invitrogen; Thermo Fisher Scientific, Inc.) according to the manufacturer's protocol. At $48 \mathrm{~h}$ post-transfection, the expression levels of HMGA2 and VEGFA were determined by reverse transcription-quantitative PCR (RT-qPCR) and western blotting.

Cell Counting Kit 8 (CCK-8) assay. A CCK-8 assay was performed to assess cell proliferation. Briefly, EH-GB1 cells or transfected cells $\left(1 \times 10^{4}\right.$ cells/well $)$ were cultured in 96-well plates for 24,48 and $72 \mathrm{~h}$ at $37^{\circ} \mathrm{C}$. At each time point, $10 \mu \mathrm{l}$ CCK-8 reagent (Beyotime Institute of Biotechnology) was added into each well and cells were incubated at $37^{\circ} \mathrm{C}$ for a further $2 \mathrm{~h}$ in the dark. The optical density of each well was measured at a wavelength of $450 \mathrm{~nm}$ using a microplate reader (Thermo Fisher Scientific, Inc.).

Wound healing assay. For the wound healing assay, $1 \times 10^{5}$ EH-GB1 cells/well were seeded into 12-well plates. Following incubation at $37^{\circ} \mathrm{C}$ for $48 \mathrm{~h}$, cells reached $90 \%$ confluence, and a scratch was drawn across the center of each well using a $200 \mu \mathrm{l}$ plastic pipette tip to generate an artificial wound. The cells were then washed three times with fresh serum-free DMEM and cultured in this medium. Following incubation at $37^{\circ} \mathrm{C}$ for $24 \mathrm{~h}$, cell migration into the wound area was visualized using a light microscope (magnification, x100; Olympus Corporation) and ImageJ v.1.8 software (National Institutes of Health) was used for quantification.

Transwell assay. Cell invasion was assessed using 24-well Transwell chambers (Corning, Inc.), which were precoated with Matrigel (Thermo Fisher Scientific, Inc.) at $37^{\circ} \mathrm{C}$ for $10 \mathrm{~h}$. Briefly, $2 \times 10^{4} \mathrm{EH}-\mathrm{GB} 1$ cells in $200 \mu \mathrm{l}$ serum-free DMEM were plated into the upper chamber, while the lower chamber was filled with $500 \mu \mathrm{l}$ normal DMEM containing 15\% FBS. After incubation at $37^{\circ} \mathrm{C}$ for $20 \mathrm{~h}$, cells were fixed with $4 \%$ paraformaldehyde for $20 \mathrm{~min}$ and stained with crystal violet for $20 \mathrm{~min}$ both at room temperature. The invasive cells were counted in three randomly selected fields of view under a light microscope (magnification, x100; Nikon Corporation) and ImageJ v.1.8 software (National Institutes of Health) was used for quantification.

Western blotting. Total protein was extracted from cells using RIPA lysis buffer (Beyotime Institute of Biotechnology) supplemented with protease inhibitors (Roche Diagnostics $\mathrm{GmbH}$ ). Total protein was quantified using a BCA assay and the protein samples (30 $\mu \mathrm{g}$ per lane) were separated via 10\% SDS-PAGE. The separated proteins were subsequently transferred onto PVDF membranes and blocked with 5\% non-fat milk for $2 \mathrm{~h}$ at room temperature. The membranes were then incubated with the following primary antibodies at $4^{\circ} \mathrm{C}$ overnight: Anti-HMGA2 (1:1,000; cat. no. ab97276; Abcam), anti-Ki67 (1:1,000; cat. no. ab16667; Abcam), anti-proliferating cell nuclear antigen (PCNA; 1:1,000; cat. no. ab18197; Abcam), anti-MMP2 (1:1,000; cat. no. ab92536; Abcam), anti-MMP9 (1:1,000; cat. no. ab76003; Abcam), anti-N-cadherin (1:1,000; cat. no. ab76011; Abcam), anti-Slug (1:1,000; cat. no. ab27568; Abcam), anti-zinc finger E-box-binding homeobox 1 (ZEB1; 1:1,000; cat. no. ab203829; 
Abcam), anti-VEGFA (1:200; cat. no. ab1316; Abcam), anti-CD31 (1:1,000; cat. no. ab9498; Abcam), anti-VEGFR1 (1:1,000; cat. no. ab32152; Abcam), anti-VEGFR2 (1:1,000; cat. no. ab134191; Abcam) or anti-GAPDH (1:1,0000; cat. no. ab181602; Abcam). Following the primary antibody incubation, the membranes were incubated with a HRP-conjugated goat anti-rabbit secondary antibody (1:5,000; cat. no. ab97080; Abcam) or rabbit anti-mouse secondary antibody (1:5,000; cat. no. ab6728; Abcam) for $2 \mathrm{~h}$ at room temperature. Protein bands were visualized using an ECL reagent (Thermo Fisher Scientific, Inc.) on a ChemiDoc XRS Imaging system (Bio-Rad Laboratories, Inc.). ImageJ v.1.8 software (National Institutes of Health) was used for semi-quantification.

$R T-q P C R$. Total RNA was extracted from cells using TRIzol ${ }^{\circledR}$ reagent (Invitrogen; Thermo Fisher Scientific, Inc.). Total RNA was reverse transcribed into cDNA using PrimeScript Reverse Transcriptase (Takara Bio, Inc.) according to the manufacturer's protocol. qPCR was subsequently performed using SYBR Green kit (Beijing Solarbio Science \& Technology Co., Ltd.) on a StepOnePlus ${ }^{\text {TM }}$ Real-Time PCR system (Applied Biosystems; Thermo Fisher Scientific, Inc.). The thermocycling conditions were as follows: $95^{\circ} \mathrm{C}$ for $15 \mathrm{~min}, 35$ cycles of $94^{\circ} \mathrm{C}$ for $1 \mathrm{~min}, 53.6^{\circ} \mathrm{C}$ for $30 \mathrm{sec}$, and $72^{\circ} \mathrm{C}$ for $30 \mathrm{sec}$, followed by a final extension at $72^{\circ} \mathrm{C}$ for $5 \mathrm{~min}$. The primer sequences used for the qPCR are listed in Table I. GAPDH was used as a reference gene and the relative gene expression levels were calculated using the $2^{-\Delta \Delta \mathrm{Cq}}$ method (24).

Tube formation assay. In vitro vascular tube formation assay was performed as previously described (25). Briefly, 96-well plates were precoated with Matrigel and incubated at $37^{\circ} \mathrm{C}$ for $1 \mathrm{~h}$. HUVECs were seeded onto Matrigel-coated 96-well plates at a density of $2 \times 10^{6}$ cells/well and cultured in the presence of DMEM from EH-GB1 cells transfected with different siRNAs/ov plasmids at $37^{\circ} \mathrm{C}$ for $24 \mathrm{~h}$. The tube formation ability of HUVECs was observed under a phase contrast light microscope (magnification, x40; MS5; Leica Microsystems, Ltd.). The number of formed tubes was quantified using ImageJ v.1.8 software (National Institutes of Health).

Bioinformatics analysis. The Search Tool for the Retrieval of Interacting Genes/Proteins (STRING; www.string-db.org) is an online database used for searching known protein interaction relationships. HMGA2 and VEGFA were entered together in multiple protein interfaces and Homo sapiens was chosen as the species, then the results were automatically displayed.

Statistical analysis. Data are presented as the mean \pm SD. All statistical analyses were performed using GraphPad Prism 8.0 software (GraphPad Software, Inc.). Statistical comparisons among multiple groups were performed using a one-way ANOVA followed by a Tukey's post hoc test. $\mathrm{P}<0.05$ was considered to indicate a statistically significant difference. All experiments were repeated at least three times.

\section{Results}

HMGA2 knockdown and silencing inhibits and promotes, respectively, GBC cell proliferation, migration, invasion
Table I. Primer sequences used for reverse transcriptionquantitative PCR.

\begin{tabular}{ll}
\hline Gene & \multicolumn{1}{c}{ Primer sequence $\left(5^{\prime} \rightarrow 3^{\prime}\right)$} \\
\hline HMGA2 & F: GCCAAGAGGCAGACCTAGGAAA \\
& R: CATGGCAATACAGAATAAGTGGTCA \\
VEGFA & F: GCCATCCAATCGAGACCCTG \\
& R: ATTAGACAGCAGCGGGCAC \\
CD31 & F: TGAGTGGTGGGCTCAGATTG \\
& R: TGAGTCTAGGTCGGGGAGTG \\
VEGFR1 & F: CTGGGCAGCAGACAAATCCT \\
& R: GCAGTGCTCACCTCTGATTGT \\
VEGFR2 & F: CGGTCAACAAAGTCGGGAGA \\
& R: CAGTGCACCACAAAGACACG \\
GAPDH & F: CAACAGCCTCAAGATCATCAGC \\
& R: TTCTAGACGGCAGGTCAGGTC \\
\hline
\end{tabular}

F, forward; R, reverse; HMGA2, high mobility group AT-hook 2.

and EMT. Firstly, HMGA2 was silenced or overexpressed in the GBC cell line, EH-GB1. As shown in Fig. 1A-D, cell transfection with siRNA-HMGA2 or ov-HMGA2 successfully downregulated and upregulated, respectively, the mRNA and protein expression levels of HMGA2 compared with the siRNA-NC and ov-NC groups, respectively. Subsequently, a CCK-8 assay was performed to evaluate cell proliferation. The results showed that HMGA2 knockdown significantly inhibited cell proliferation at $72 \mathrm{~h}$ post-transfection compared with the siRNA-NC group, while HMGA2 overexpression significantly promoted cell proliferation at 48 and $72 \mathrm{~h}$ post-transfection compared with the ov-NC group (Fig. 1E). Consistent with these findings, the protein expression levels of Ki67 and PCNA were significantly downregulated following HMGA2 silencing compared with the siRNA-NC group, while the opposite effect was observed following HMGA2 overexpression compared with the ov-NC group (Fig. 1F).

Wound healing and Transwell assays were used to evaluate the cell migratory and invasive abilities, respectively. As shown in Fig. 2A and B, cell migration and invasion were significantly inhibited upon HMGA2 silencing compared with the siRNA-NC group, while both migration and invasion were significantly increased following HMGA2 overexpression compared with the ov-NC group. Consistent with these results, the expression levels of cell migration- and invasion-related proteins, namely MMP2 and MMP9, were significantly downregulated by HMGA2 silencing compared with the siRNA-NC group. The opposite effect was observed following HMGA2 overexpression compared with the ov-NC group (Fig. 3A and B). The expression levels of the EMT-related proteins, N-cadherin, Slug and ZEB1, were also significantly upregulated and downregulated following transfection of EH-GB1 cells with ov-HMGA2 or siRNA-HMGA2, respectively, compared with the respective NCs (Fig. 3C). The aforementioned findings suggested that HMGA2 may play a promotive role in the proliferation, migration, invasion and EMT of GBC cells. 
A

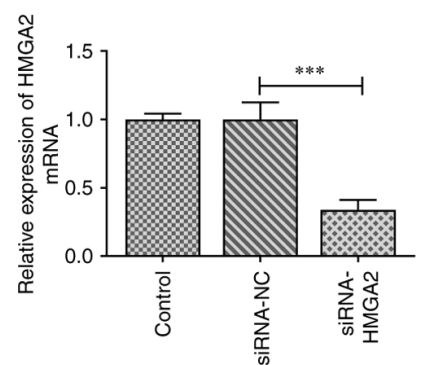

C

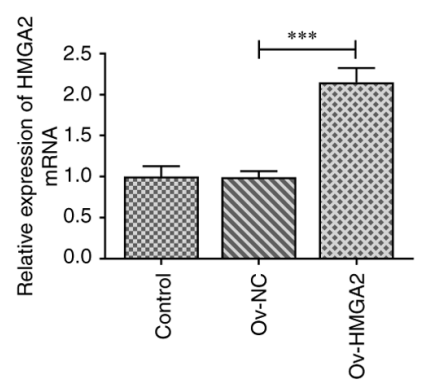

E

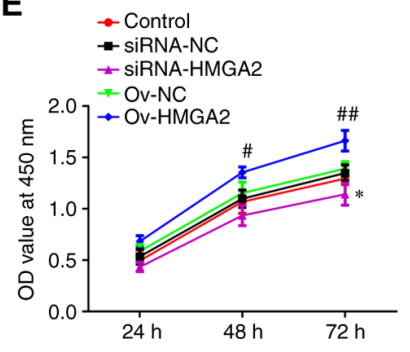

B

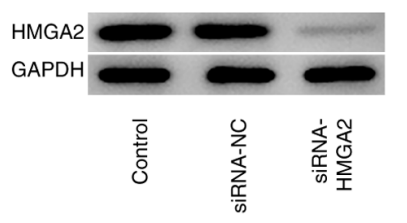

D

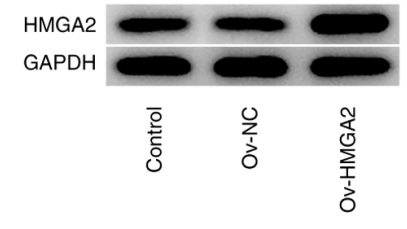

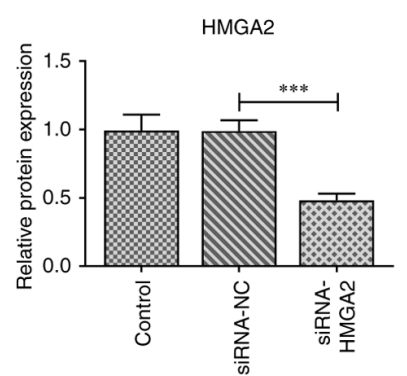

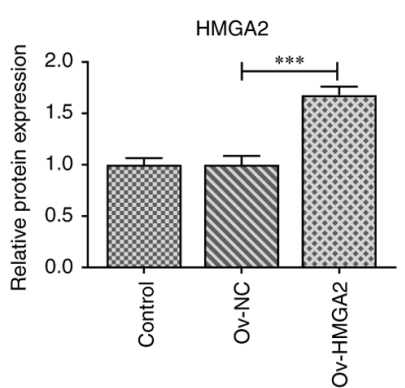

$\mathbf{F}$

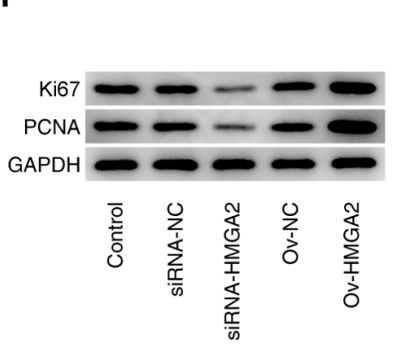

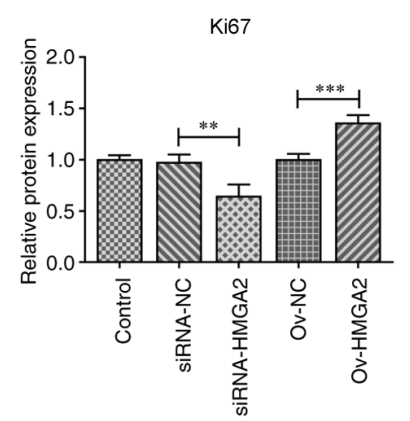

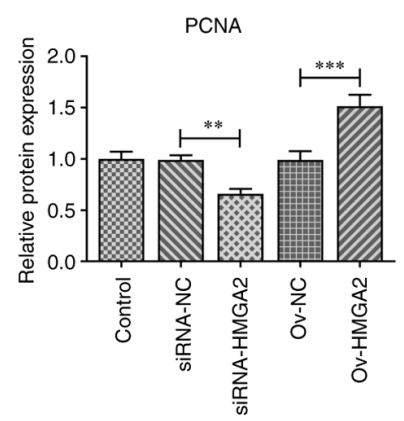

Figure 1. Effects of HMGA2 overexpression and knockdown on gallbladder cancer cell proliferation. (A) mRNA and (B) protein expression levels of HMGA2 in EH-GB1 cells following HMGA2 knockdown. (C) mRNA and (D) protein expression levels of HMGA2 in EH-GB1 cells following HMGA2 overexpression. ${ }^{* * *} \mathrm{P}<0.001$. (E) Proliferation of EH-GB1 cells transfected with the indicated plasmids or siRNAs was assessed at 24, 48 and $72 \mathrm{~h}$ post-transfection using a Cell Counting Kit-8 assay. ${ }^{\mathrm{P}} \mathrm{P}<0.05$ vs. si-NC; ${ }^{\# \mathrm{P}}<0.05$, ${ }^{\sharp \#} \mathrm{P}<0.01$ vs. ov-NC. (F) Protein expression levels of Ki67 and PCNA in EH-GB1 cells were determined using western blotting. ${ }^{* *} \mathrm{P}<0.01,{ }^{* * *} \mathrm{P}<0.001$. HMGA2, high mobility group AT-hook 2 ; siRNA, small interfering RNA; ov-, overexpression; PCNA, proliferating cell nuclear antigen; $\mathrm{OD}$, optical density; $\mathrm{NC}$, negative control.

HMGA2 knockdown downregulates, while HMGA2 overexpression upregulates, VEGFA expression in GBC cells. Bioinformatics analysis using the STRING database predicted that HMGA2 could interact with VEGFA (Fig. 4A). Subsequently, the expression levels of VEGFA were determined in EH-GB1 cells following HMGA2 knockdown or overexpression. The results showed that both the mRNA and protein expression levels of VEGFA were downregulated by HMGA2 knockdown compared with the siRNA-NC group, while the opposite effect was observed upon HMGA2 overexpression compared with the ov-NC group (Fig. 4B and C). GBC cells were transfected with ov-VEGFA plasmid, and the results demonstrated that the expression level VEGFA was upregulated in the ov-VEGFA group compared with the ov-NC group (Fig. 4D). These data indicated that HMGA2 may positively regulate the expression of VEGFA.

VEGFA overexpression abrogates the inhibitory effect of HMGA2 silencing on HUVEC tube formation and expression of CD31, VEGFR1 and VEGFR2. It is well known that VEGFA can induce tumor angiogenesis (26). Therefore, GBC cells transfected with siRNA-HMGA2 were transfected with an ov-VEGFA plasmid and the culture medium was then used to stimulate HUVECs. Subsequently, the tube formation ability of HUVECs and the expression levels of CD31, VEGFR1 and VEGFR2 were evaluated. As shown in Fig. 5A, HMGA2 silencing significantly attenuated the tube formation ability of HUVECs compared with the siRNA-NC group. However, the co-transfection with ov-VEGFA partially rescued the tube formation ability of HUVECs transfected with siRNA-HMAG2. In addition, both the mRNA and protein expression levels of CD31, VEGFR1 and VEGFR2 were markedly downregulated in HUVECs following HMGA2 silencing compared with the siRNA-NC group. However, the aforementioned effect was partially rescued by VEGFA overexpression (Fig. 5B and C). These results suggested that HMGA2 knockdown may inhibit the angiogenesis of HUVECs, possibly via downregulating VEGFA expression.

\section{Discussion}

The HMGA2 protein belongs to the HMGA subfamily of HMG proteins and encodes a 108 amino acid protein. HMGA2 

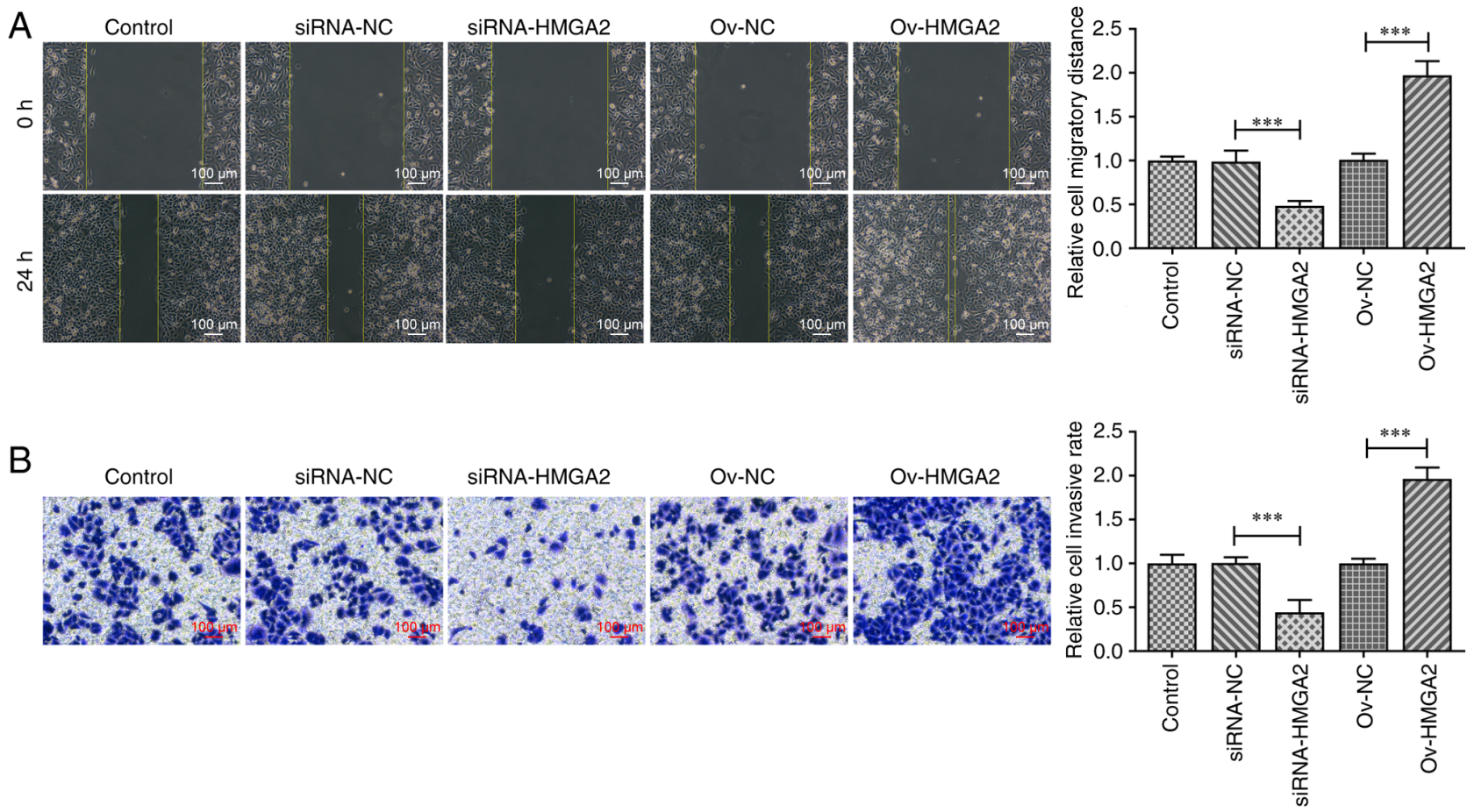

Figure 2. Effects of HMGA2 overexpression and knockdown on gallbladder cancer cell migration and invasion. Representative images and semi-quantitative analysis for (A) wound healing and (B) Transwell assays of EH-GB1 cells in different treatment groups. Scale bar, $100 \mu \mathrm{m} .{ }^{* * * *} \mathrm{P}<0.001$. HMGA2, high mobility group AT-hook 2; siRNA, small interfering RNA; NC, negative control; ov-, overexpression.

A

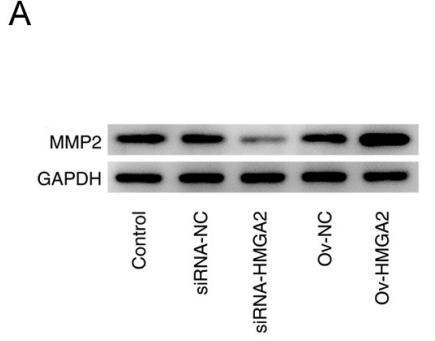

C

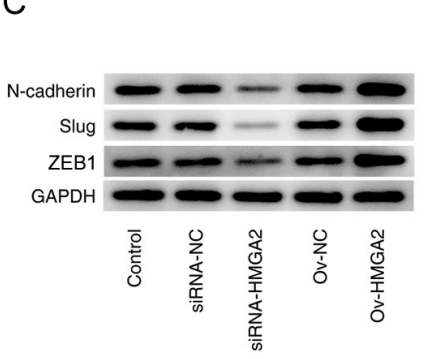

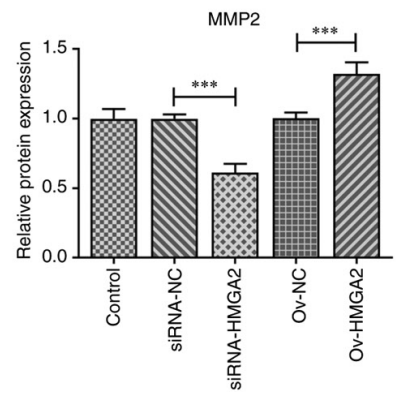

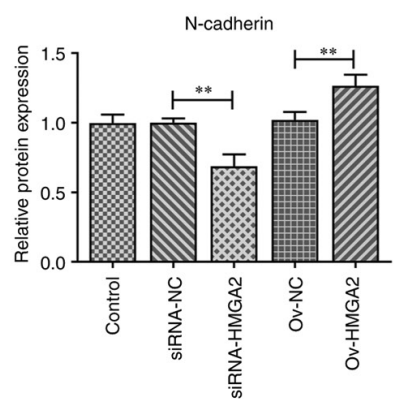

B
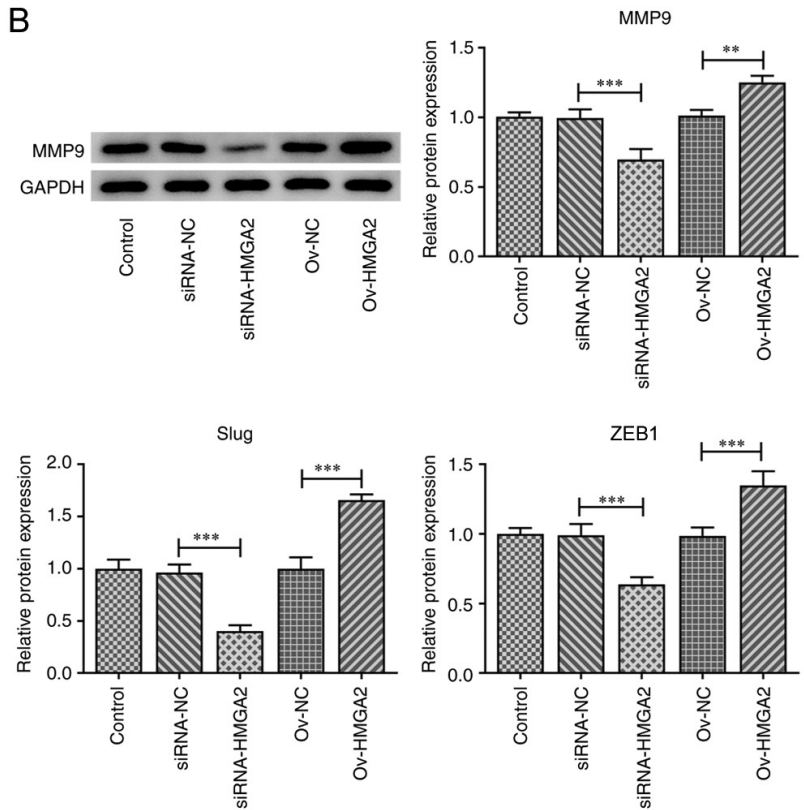

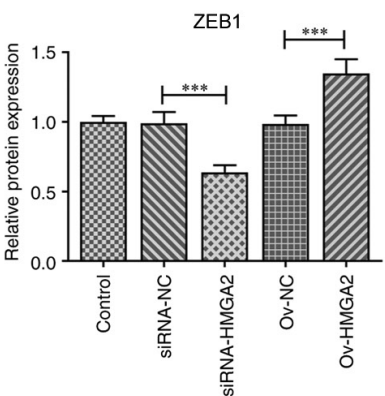

Figure 3. Effects of HMGA2 overexpression and knockdown on the epithelial-mesenchymal transition of gallbladder cancer cells. Protein expression levels of (A) MMP2, (B) MMP9, and (C) N-cadherin, Slug and ZEB1 in EH-GB1 cells transfected with the indicated plasmids or siRNAs were detected using western blotting. ${ }^{* *} \mathrm{P}<0.01,{ }^{* * *} \mathrm{P}<0.001$. HMGA2, high mobility group AT-hook 2; ZEB1, zinc finger E-box-binding homeobox 1; Slug, snail family transcriptional repressor 2; siRNA, small interfering RNA; NC, negative control; ov-, overexpression.

is a small, non-histone, chromatin-associated protein with no intrinsic transcriptional activity (27). However, it can modulate gene transcription via altering chromatin architecture, thereby enhancing or suppressing the transcriptional activity of several human genes, eventually affecting a variety of biological processes (28). It has been reported that HMGA2 is upregulated in numerous types of human cancer, indicating that it may serve a crucial role in cancer development and carcinogenesis (10). Emerging evidence has suggested that HMGA2 played an important role in the majority of human cancer types, including lung (16), pancreatic (29), colorectal (15) and breast (30) cancer, via regulating the cell 
A

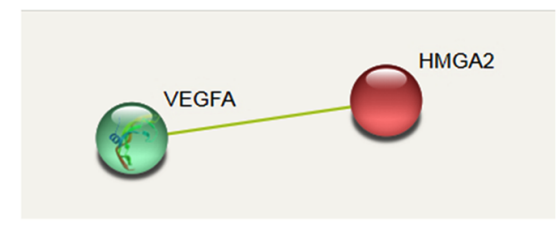

C

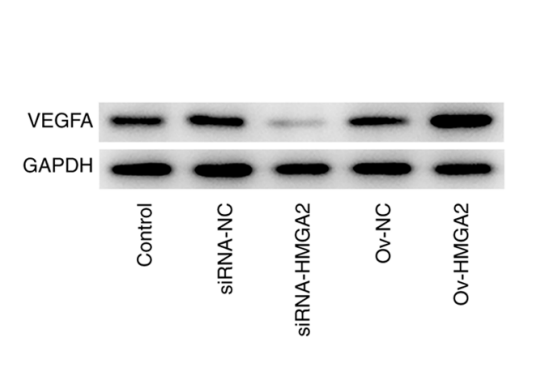

B

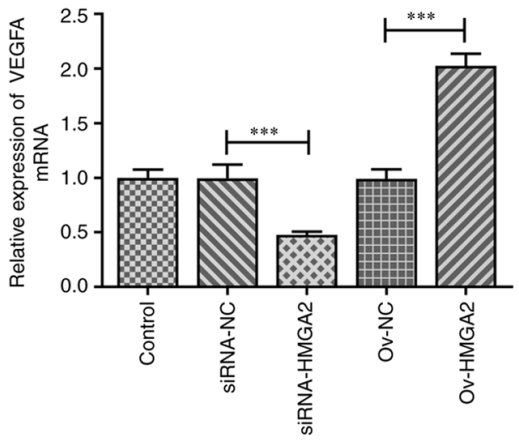

VEGFA

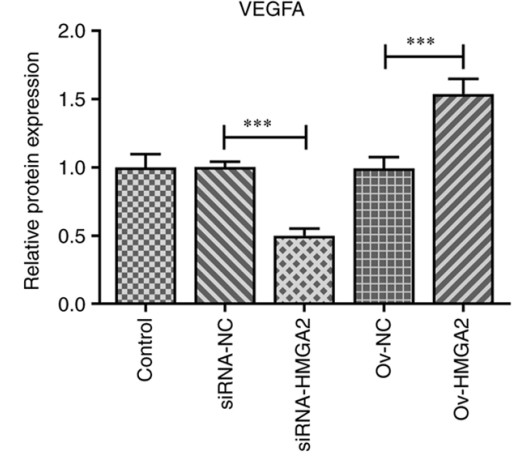

D

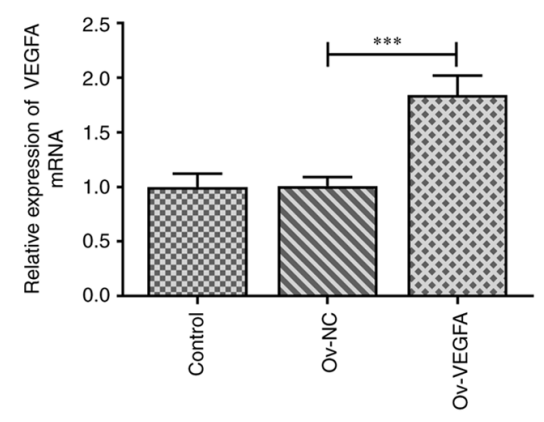

Figure 4. Association between HMGA2 and VEGFA. (A) Interaction between VEGFA and HMGA2 was predicted using the Search Tool for the Retrieval of Interacting Genes/Proteins database. (B) mRNA and (C) protein expression levels of VEGFA in EH-GB1 cells following HMGA2 silencing or overexpression. (D) mRNA expression levels of VEGFA in EH-GB1 cells following VEGFA overexpression. ${ }^{* * * *} \mathrm{P}<0.001$. HMGA2, high mobility group AT-hook 2; siRNA, small interfering RNA; NC, negative control; ov-, overexpression.

cycle, apoptosis, DNA damage repair and cell senescence, as well as promoting EMT and maintaining telomere length (10). The results of the present study showed that HMGA2 could promote GBC cell proliferation, migration, invasion and EMT. HMGA2 is normally expressed in mesenchymal cells and EH-GB1 is a metastatic gallbladder cancer cell line, in which E-cadherin is expressed at low levels (31). When epithelial cells undergo EMT, they adopt a mesenchymal cell phenotype to promote metastasis (32). The results of the present study revealed that HMGA2 knockdown effectively inhibited the aforementioned cellular processes in GBC cells. Furthermore, HMGA2 silencing also suppressed angiogenesis in HUVECs via targeting VEGFA.

Ki67 and PCNA are the most commonly used cell proliferation markers (33). Moreover, the increased expression of both markers is associated with the active proliferation of tumor cells (34). In the present study, HMGA2 knockdown and overexpression downregulated and upregulated, respectively, the expression levels of both Ki67 and PCNA, suggesting that HMGA2 may promote GBC cell proliferation. The invasion and metastasis of malignant tumors has been discovered to contribute to chemotherapy failure and death in patients with cancer (35). The results of the wound healing and Transwell assays in the current study revealed that HMGA2 silencing attenuated the migratory and invasive abilities of GBC cells, whereas HMGA2 overexpression exhibited the opposite results.

MMP2 and MMP9 belong to the MMP family and are responsible for degrading the extracellular matrix, thus accelerating tumor cell migration, invasion and angiogenesis (36). Herein, the protein expression levels of MMP2 and MMP9 were found to be downregulated following HMGA2 knockdown and upregulated following HMGA2 overexpression. Furthermore, the same results were observed when the expression levels of N-cadherin, Slug and ZEB1 were determined. The upregulated expression levels of $\mathrm{N}$-cadherin, Slug and ZEB1 are typical features of EMT, and represents one of the key steps required for the invasion and metastasis of malignant tumors of epithelial origin (37). Collectively, these data suggested that HMGA2 may promote GBC cell migration and invasion.

The growth of solid tumors is accompanied by the induction of angiogenesis and VEGFA is considered as a key regulator of this processes (38). Angiogenesis is a biological process that leads to the formation of new blood vessels from pre-existing blood vessels (39). Tumor cells produce or cause the microenvironment to generate pro-angiogenic signals, which can recruit and expand endothelial cells (40). In addition, a retrospective review reported that VEGFA was expressed in $\sim 80 \%$ of GBCs, and $56.3 \%$ of the 84 patients had high expression levels of VEGFA, which has been found to be an independent prognostic factor for survival in GBC (41). Notably, the current study demonstrated that HMGA2 could bind with VEGFA to positively regulate its expression. Therefore, it was hypothesized that HMGA2 may modulate angiogenesis via targeting VEGFA. The present study co-transfected GBC cells with siRNA-HMGA2 and ov-VEGFA and the CM was collected to stimulate HUVECs. Consistent with the aforementioned hypothesis, HMGA2 silencing significantly attenuated the tube formation ability of HUVECs. Consistently, the mRNA and protein expression levels of CD31, VEGFR1 and VEGFR2 were also downregulated following HMGA2 knockdown. The 
A

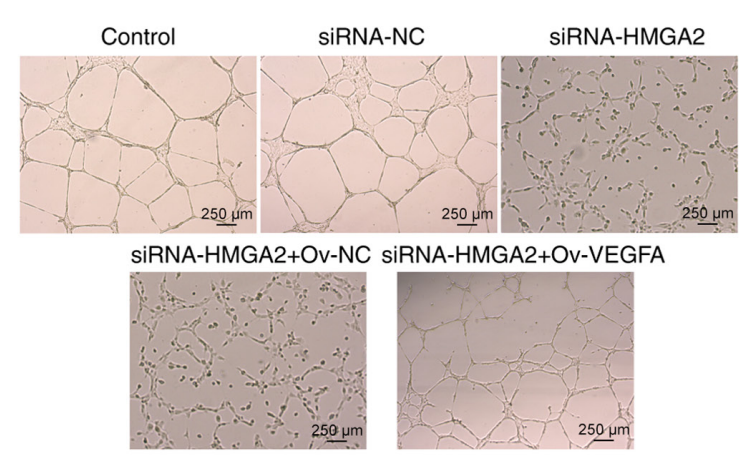

B

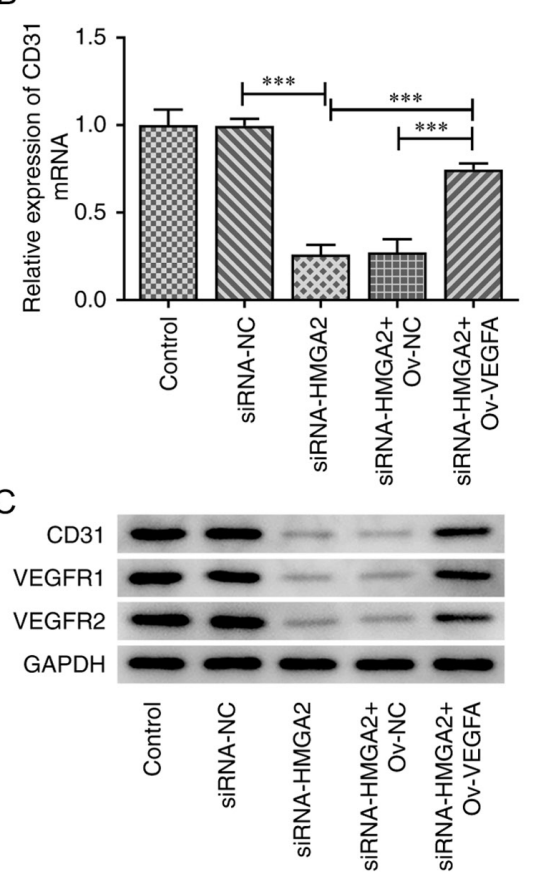

CD31

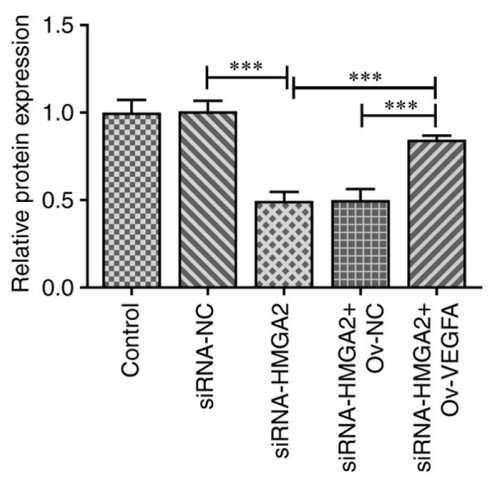

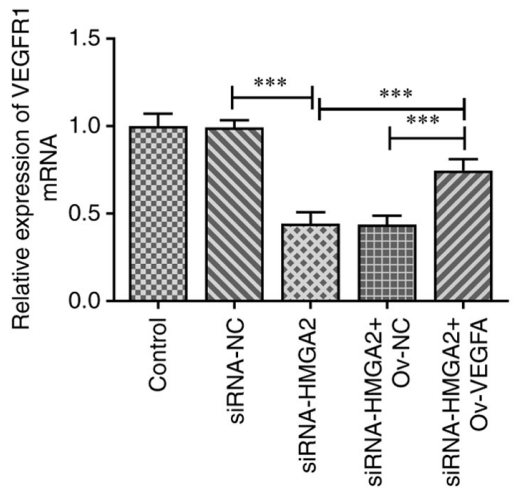
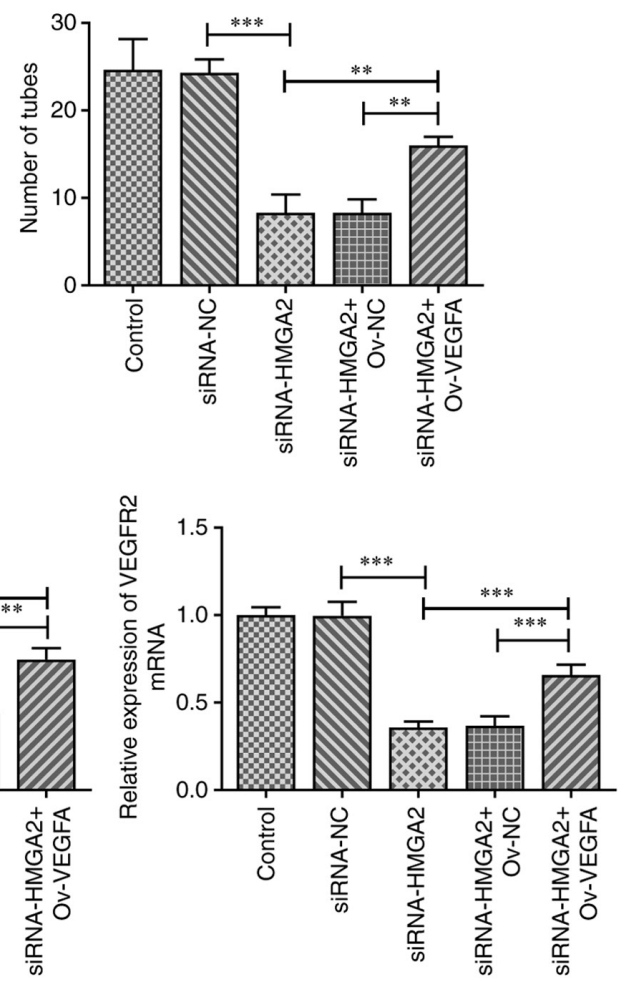

VEGFR1

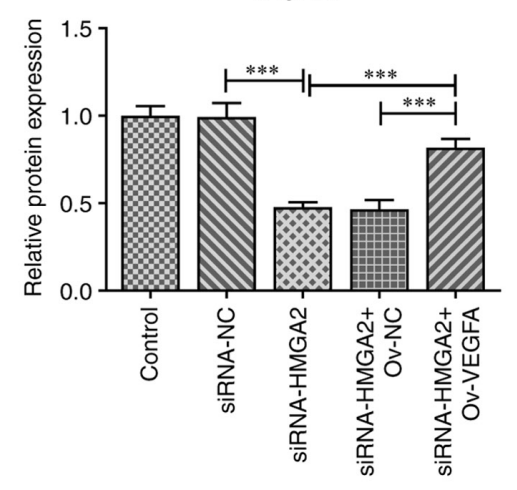

VEGFR2

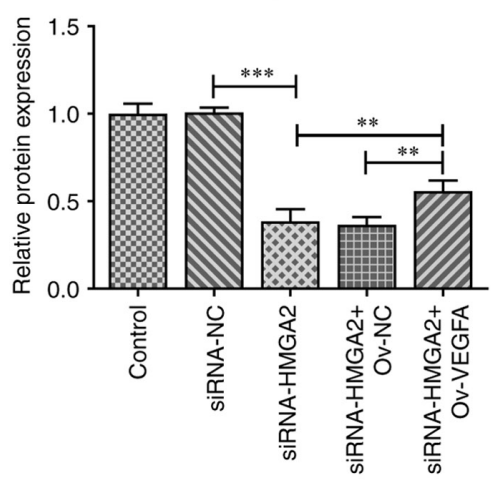

Figure 5. Effects of HMGA2 silencing and VEGFA overexpression on angiogenesis. (A) Representative images and semi-quantitative analysis of the tube formation ability of HUVECs cultured in the presence of culture medium obtained from EH-GB1 cells in different treatment groups. Scale bar, $250 \mu$ m. (B) mRNA and (C) protein expression levels of CD31, VEGFR1 and VEGFR2 in HUVECs stimulated with culture medium obtained from EH-GB1 cells in different treatment groups. ${ }^{* *} \mathrm{P}<0.01,{ }^{* * *} \mathrm{P}<0.001$. HMGA2, high mobility group AT-hook 2; siRNA, small interfering RNA; NC, negative control; ov-, overexpression.

expression of CD31, also known as platelet endothelial cell adhesion molecule 1 , is often used to evaluate tumor angiogenesis (42). VEGFA is activated after its binding to VEGFR1 and VEGFR2, two receptors involved in angiogenesis-related signaling pathways (43). Herein, the results suggested that HMGA2 may be involved in the angiogenesis of GBC cells. Furthermore, the overexpression of VEGFA partially abrogated the inhibitory effect of HMGA2 silencing on angiogenesis, thus verifying that HMGA2 may regulate angiogenesis via modulating VEGFA expression. However, the analysis of MMP activation in the present study was limited to western blotting, and gelatin zymography and RT-qPCR are required for a more comprehensive analysis of MMP activation in the future. In addition, the present study only used in vitro cell models to generate the results; therefore, future studies should focus on verifying the results of the current study using in vivo 
models to determine the potential mechanisms involved in the effects of HMGA2.

In conclusion, the results of the present study suggested that the overexpression of HMGA2 in GBC cells may promote cancer progression by inducing cell migration, invasion and angiogenesis. Therefore, HMGA2 may serve as a predictive factor in GBC and targeting HMGA2 could be considered as a potential therapeutic approach for GBC.

\section{Acknowledgements}

Not applicable.

\section{Funding}

No funding was received.

\section{Availability of data and materials}

The datasets used and/or analyzed during the current study are available from the corresponding author on reasonable request.

\section{Author's contributions}

JY and YZ conceived and designed the present study, and JY was involved in drafting the manuscript. JY, PD and $\mathrm{XQ}$ conducted the experiments. XQ and $\mathrm{YH}$ performed the data analysis. YZ performed the bioinformatics analysis and provided critical comments on the revision of the manuscript. All authors read and approved the final manuscript. JY and YZ confirm the authenticity of all the raw data.

\section{Ethics approval and consent to participate}

Not applicable.

\section{Patient consent for publication}

Not applicable.

\section{Competing interests}

The authors declare that they have no competing interests.

\section{References}

1. Zhou F, Zhang Y, Sun J and Yang X: Characteristics of a novel cell line ZJU-0430 established from human gallbladder carcinoma. Cancer Cell Int 19: 190, 2019.

2. Ramachandran A, Srivastava DN and Madhusudhan KS: Gallbladder cancer revisited: The evolving role of a radiologist. Br J Radiol 94: 20200726, 2021.

3. Hickman L and Contreras C: Gallbladder cancer: Diagnosis, surgical management, and adjuvant therapies. Surg Clin North Am 99: 337-355, 2019.

4. Brandi G, Venturi M, Pantaleo MA and Ercolani G: Cholangiocarcinoma: Current opinion on clinical practice diagnostic and therapeutic algorithms: A review of the literature and a long-standing experience of a referral center. Digestive Liver Dis 48: 231-241, 2016.

5. Siegel RL, Fedewa SA, Miller KD, Goding-Sauer A, Pinheiro PS, Martinez-Tyson D and Jemal A: Cancer statistics for Hispanics/Latinos, 2015. CA Cancer J Clin 65: 457-480, 2015.
6. Goetze TO: Gallbladder carcinoma: Prognostic factors and therapeutic options. World J Gastroenterol 21: 12211-12217, 2015.

7. Jiang W, Zhao B, Li Y, Qi D and Wang D: Modification of the 8th American joint committee on cancer staging system for gallbladder carcinoma to improve prognostic precision. BMC Cancer 20: 1129, 2020.

8. Sharma A, Sharma KL, Gupta A, Yadav A and Kumar A: Gallbladder cancer epidemiology, pathogenesis and molecular genetics: Recent update. World J Gastroenterol 23: 3978-3998, 2017.

9. Sternby Eilard M, Lundgren L, Cahlin C, Strandell A, Svanberg T and Sandström P: Surgical treatment for gallbladder cancer-a systematic literature review. Scand J Gastroenterol 52: 505-514, 2017.

10. Zhang S, Mo Q and Wang X: Oncological role of HMGA2 (Review). Int J Oncol 55: 775-788, 2019.

11. Li Y, Zhao Z, Xu C, Zhou Z, Zhu Z and You T: HMGA2 induces transcription factor Slug expression to promote epithelial-to-mesenchymal transition and contributes to colon cancer progression. Cancer Lett 355: 130-140, 2014.

12. Lovnicki J, Gan Y, Feng T, Li Y, Xie N, Ho CH, Lee AR, Chen X, Nappi L, Han B, et al: LIN28B promotes the development of neuroendocrine prostate cancer. J Clin Invest 130: 5338-5348, 2020.

13. Sun J, Sun B, Sun R, Zhu D, Zhao X, Zhang Y, Dong X, Che N, $\mathrm{Li}$, J, Liu F, et al: HMGA2 promotes vasculogenic mimicry and tumor aggressiveness by upregulating Twist1 in gastric carcinoma. Sci Rep 7: 2229, 2017.

14. Fedele M, Palmieri D and Fusco A: HMGA2: A pituitary tumour subtype-specific oncogene? Mol Cell Endocrinol 326: 19-24, 2010.

15. Wang $\mathrm{X}$, Wang $\mathrm{J}$ and $\mathrm{Wu} \mathrm{J}$ : Emerging roles for HMGA2 in colorectal cancer. Transl Oncol 14: 100894, 2021.

16. Gao X, Dai M, Li Q, Wang Z, Lu Y and Song Z: HMGA2 regulates lung cancer proliferation and metastasis. Thorac Cancer 8: 501-510, 2017.

17. Hombach-Klonisch S, Kalantari F, Medapati MR, Natarajan S, Krishnan SN, Kumar-Kanojia A, Thanasupawat T, Begum F, $\mathrm{Xu}$ FY, Hatch GM, et al: HMGA2 as a functional antagonist of PARP1 inhibitors in tumor cells. Mol Oncol 13: 153-170, 2019.

18. Zou Q, Xiong L, Yang Z, Lv F, Yang L and Miao X: Expression levels of HMGA2 and CD9 and its clinicopathological significances in the benign and malignant lesions of the gallbladder. World J Surg Oncol 10: 92, 2012.

19. Zhou H, Guo W, Zhao Y, Wang Y, Zha R, Ding J, Liang L, Hu J, Shen H, Chen Z, et al: MicroRNA-26a acts as a tumor suppressor inhibiting gallbladder cancer cell proliferation by directly targeting HMGA2. Int J Oncol 44: 2050-2058, 2014.

20. Yuan L, Guo F, Wang L and Zou Q: Prediction of tumor metastasis from sequencing data in the era of genome sequencing. Brief Funct Genomics 18: 412-418, 2019.

21. Scully OJ, Bay BH, Yip G and Yu Y: Breast cancer metastasis. Cancer Genomics Proteomics 9: 311-320, 2012.

22. Steeg PS: Targeting metastasis. Nat Rev Cancer 16: 201-218, 2016.

23. Viallard $C$ and Larrivée B: Tumor angiogenesis and vascular normalization: Alternative therapeutic targets. Angiogenesis 20: 409-426, 2017.

24. Livak KJ and Schmittgen TD: Analysis of relative gene expression data using real-time quantitative PCR and the 2(-Delta Delta C(T)) method. Methods 25: 402-408, 2001.

25. Zhang JT, Fan YZ, Chen CQ, Zhao ZM and Sun W: Norcantharidin: A potential antiangiogenic agent for gallbladder cancers in vitro and in vivo. Int J Oncol 40: 1501-1514, 2012.

26. Li X, Hu Z, Shi H, Wang C, Lei J and Cheng Y: Inhibition of VEGFA increases the sensitivity of ovarian cancer cells to chemotherapy by suppressing VEGFA-mediated autophagy. Onco Targets Ther 13: 8161-8171, 2020.

27. Su L, Deng Z and Leng F: The mammalian high mobility group protein AT-Hook 2 (HMGA2): Biochemical and biophysical properties, and its association with adipogenesis. Int J Mol Sci 21: 3710, 2020.

28. Wu J and Wei JJ: HMGA2 and high-grade serous ovarian carcinoma. J Mol Med (Berl) 91: 1155-1165, 2013.

29. Chiou SH, Dorsch M, Kusch E, Naranjo S, Kozak MM, Koong AC, Winslow MM and Grüner BM: Hmga2 is dispensable for pancreatic cancer development, metastasis, and therapy resistance. Sci Rep 8: 14008, 2018.

30. Zhao W, Geng D, Li S, Chen Z and Sun M: LncRNA HOTAIR influences cell growth, migration, invasion, and apoptosis via the miR-20a-5p/HMGA2 axis in breast cancer. Cancer Med 7: 842-855, 2018 . 
31. Hao J, Yang Z, Wang L, Zhang Y, Shu Y, Jiang L, Hu Y, Lv W, Dong P and Liu Y: Downregulation of BRD4 inhibits gallbladder cancer proliferation and metastasis and induces apoptosis via PI3K/AKT pathway. Int J Oncol 51: 823-831, 2017.

32. Shintani Y, Maeda M, Chaika N, Johnson KR and Wheelock MJ: Collagen I promotes epithelial-to-mesenchymal transition in lung cancer cells via transforming growth factor-beta signaling. Am J Respir Cell Mol Biol 38: 95-104, 2008.

33. Ruan Y, Wang L and Lu Y: HDAC6 inhibitor, ACY1215 suppress the proliferation and induce apoptosis of gallbladder cancer cells and increased the chemotherapy effect of gemcitabine and oxaliplatin. Drug Dev Res 82: 598-604, 2021.

34. Juríková M, Danihel L, Polák S and Varga I: Ki67, PCNA, and MCM proteins: Markers of proliferation in the diagnosis of breast cancer. Acta Histochem 118: 544-552, 2016.

35. Nowakowska A and Tarasiuk J: Invasion and metastasis of tumour cells resistant to chemotherapy. Postepy Hig Med Dosw (Online) 71: 380-397, 2017.

36. Pittayapruek P, Meephansan J, Prapapan O, Komine M and Ohtsuki M: Role of matrix metalloproteinases in photoaging and photocarcinogenesis. Int J Mol Sci 17: 668, 2016.

37. Zhou P, Wang C, Hu Z, Chen W, Qi W and Li A: Genistein induces apoptosis of colon cancer cells by reversal of epithe-

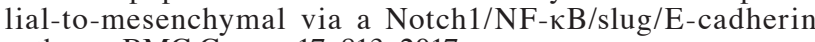
pathway. BMC Cancer 17: 813, 2017.
38. Claesson-Welsh L and Welsh M: VEGFA and tumour angiogenesis. J Intern Med 273: 114-127, 2013.

39. Simone V, Brunetti O, Lupo L, Testini M, Maiorano E, Simone M, Longo V, Rolfo C, Peeters M, Scarpa A, et al: Targeting angiogenesis in biliary tract cancers: An open option. Int J Mol Sci 18: 418, 2017.

40. Bergers $\mathrm{G}$ and Benjamin LE: Tumorigenesis and the angiogenic switch. Nat Rev Cancer 3: 401-410, 2003.

41. Sun XN, Cao WG, Wang X, Wang Q, Gu BX, Yang QC, Hu JB, Liu $\mathrm{H}$ and Zheng S: Prognostic impact of vascular endothelial growth factor-A expression in resected gallbladder carcinoma. Tumour Biol 32: 1183-1190, 2011.

42. Lertkiatmongkol P, Liao D, Mei H, Hu Y and Newman PJ: Endothelial functions of platelet/endothelial cell adhesion molecule-1 (CD31). Curr Opin Hematol 23: 253-259, 2016.

43. Freire Valls A, Knipper K, Giannakouri E, Sarachaga V, Hinterkopf S, Wuehrl M, Shen Y, Radhakrishnan P, Klose J, Ulrich A, et al: VEGFR1(+) metastasis-associated macrophages contribute to metastatic angiogenesis and influence colorectal cancer patient outcome. Clin Cancer Res 25: 5674-5685, 2019.

This work is licensed under a Creative Commons Attribution-NonCommercial-NoDerivatives 4.0 International (CC BY-NC-ND 4.0) License. 